\title{
CAN FLUORINE FORM TRIPLE BONDS?
}

\author{
A PREPRINT \\ Julian M. Kleber ${ }^{*}$ \\ Freie Universität Berlin \\ Königin-Luise-Straße 2-4, 14195 Berlin \\ julian.kleber@fu-berlin.de
}

February 24, 2022

\begin{abstract}
A central dogma is that fluorine is the most electronegative element in the PSE and would thus act as a pure electron acceptor. However, to some chemists it is known, that fluorine can bond in duality. This means fluorine can act as a donor and an acceptor of bonds and thus electrons. The donor/acceptor properties of fluorine are known from the NF molecule, where a partial double bond character is present[1]. Up to now, however, chemists are not aware that fluorine may form even triple bonds, something that other elements from the second row of the PSE do as well. The subsequent work investigates theoretically the possibility of triple bonds between fluorine and $\mathrm{Mn}$ in a hetero dimer MnF. Moreover, mono fluorides isoelectronic to $\mathrm{MnF}$ and higher oxidation states of $\mathrm{MnF}$ are investigated. Furthermore, the tendency to form singlets and possibly multi-bonded states is investigated for higher transition metals and halogens as well. It is concluded that first the naive methodology of using Wigner-Witmer rules may find their limit in the case of $\mathrm{MnF}$ and isoelectronic compounds as well as higher homologues. Secondly, it is demonstrated that using ECP may lead to misleading results on higher transition metals when calculating heterodimers with halogens.
\end{abstract}

Keywords Quantum chemistry, chemical bonding, fluorine, transition metals, multi reference, Hartree-Fock, theoretical chemistry

\section{Introduction}

Computational and theoretical chemistry serve as disciplines and tools for a chemist to understand the nature of binding. Often taught qualitatively to chemist, the accurate description of a chemical bond is not straightforward even with sophisticated computers. The nature of the approximations applied in quantum chemical programs decide about the obtained results. As the knowledge in the theoretical field grows and grows, so does the experimental data available. Moreover, the field of computational chemistry becomes more and more separated from experimental chemistry. The separation of disciplines is necessary but at the same time faulty because the central theme of interest to each and every chemist is the chemical bond. However, empirical lab chemists do not have the time to deep dive into theoretical chemistry to try to understand what they are doing. Vice versa, theoretical chemists seldomly have the time to carry out a lot of experiments. Moreover, lab chemists often rely on simplified models that explain many but not close to all phenomena they will experience in their lab work. Thus, it might happen, that common rules of thumb are not questioned, and bonding models become a believed truth that then fails to explain the reality a chemist is experiencing. This work aims to use computational chemistry to explain the bonding situation in MnF.

\section{Related Work}

Riedel et al. published an article on the matrix isolation experiment to measure the IR-frequency of MnF and the state of the molecule was anticipated to be a ${ }^{7} \Sigma$ state[2]. Furthermore, an older work suggested similar high-spin states for

\footnotetext{
${ }^{*}$ Master Student at Freie Universität Berlin
} 
Table 1: Comparison of different levels of theory based on UHF calculation for different multiplicities $(2 S+1)$ and $\Sigma$ states. Energies are given in Hartree. IRREPs are given according to the Molpro manual[8].

\begin{tabular}{llllll}
$\mathrm{R}(\AA)$ & $\mathrm{HF}$ & $\mathrm{CASSCF}$ & MRCI & IRREP & Mult \\
\hline 1.86563091 & -1249.370195 & -1249.362607 & -1249.617938 & 1 & 7 \\
1.76660973 & -1249.217022 & -1249.355452 & -1249.585352 & 1 & 5 \\
- & - & & & 1 & 3 \\
1.858631860 & -1249.028371 & -1249.392305 & -1249.412418 & 1 & 1 \\
3.98585916 & -1249.126028 & & & 4 & 7 \\
1.792797860 & -1249.212249 & & & 4 & 5 \\
1.80542986 & -1249.128642 & & & 4 & 3 \\
- & - & & & 4 & 1 \\
\hline
\end{tabular}

Table 2: Comparison of different levels of theory based on RHF calculation for different multiplicities $(2 S+1)$ and $\Sigma$ states. Energies are given in Hartree. IRREPs are given according to the Molpro manual[8].

\begin{tabular}{llllll}
$\mathrm{R}(\AA)$ & $\mathrm{HF}$ & $\mathrm{CASSCF}$ & MRCI & IRREP & Mult \\
\hline 1.86828998 & -1249.368852 & -1249.392614 & 1 & 7 \\
1.88376701 & -1249.214203 & -1249.358136 & 1 & 5 \\
- & - & & 1 & 3 \\
1.85863061 & -1249.028371 & -1249.129454 & 1 & 1 \\
1.76857248 & -1249.075312 & & 4 & 7 \\
1.79656241 & -1249.206475 & & 4 & 5 \\
1.82053763 & -1249.054167 & & 4 & 3 \\
- & - & & 4 & 1 \\
\hline
\end{tabular}

transition metal fluorides[3]. The ${ }^{7} \Sigma$ however, would depict a non-boned state and can't thus be the true state of the measured MnF molecule. In the MnF there must be a bond present because an IR stretching frequency was measured.

\section{Methods}

From quantum mechanics, it is known that ROHF and UHF converge to a singlet state where appropriate. Thus, the calculations on the molecules were performed at UHF, ROHF level of theory with the cc-pVDZ basis set. Subsequent CASSCF and MRCI calculations were performed using the optimized geometries from the UHF and ROHF calculations. Orbital visualisations were done using the cc-pVTZ basis set. Dissociation curves were calculated, and vibrational analysis was done to compare the states to the measured IR curve. In addition, to provoke donor properties of F, $\mathrm{Mn}(\mathrm{IV})$ is investigated as well. Moreover, to have a higher triple bond and less ionic character, a higher charged and more electron rich transition metal were investigated. Elements with a higher tendency to form triple bonds to fluorine could for example be $\mathrm{Ni}(\mathrm{III}) \mathrm{F}$ or $\mathrm{Pd}(\mathrm{III}) \mathrm{F}$. Therefore, Ni(III)F and Pd(III)F were investigated, too. All multi-reference calculations were performed on the CURTA cluster of the Freie Universität Berlin[4] using MOLPRO[5. 6]. Visualisations were done with MOLDEN[7].

\section{Results}

The MnF immediately converged to a ${ }^{1} \Sigma$ state for the RHF and UHF calculations with no spin contamination present. The results of the calculations are summarized in Table 1 and 2 . This means that there is most likely, although not necessarily, a closed shell state. The orbital occupancies show strong ionic character of the bond, but clearly ligand field splitting and some interaction with the fluorine orbitals in the coefficients.

Plain $\mathrm{MnF}$ in the ${ }^{1} \Sigma$ state, a bond seems to be indeed present, even though it is weak. The orbital shown in compare Figure 1 is most likely to represent the weak single bond. The weakly bonding orbital is not representing the HOMO. However, the bond is rather of a single bond character and no triple bond is present. In the case of $\mathrm{MnF}$, fluorine accepts electrons into the $\mathrm{p}_{\mathrm{z}}$ orbital, but does not donate many electrons $\mathrm{p}_{\mathrm{x}}$ and $\mathrm{p}_{\mathrm{y}}$ orbitals to form a bond. Still, some interaction between the orbitals of the metal centre and ligand is present.

$\mathrm{MnF}$ in the ${ }^{7} \Sigma$ state does not show a bonding molecular orbital and, as expected, the ligand field splitting is coherent with the model. 


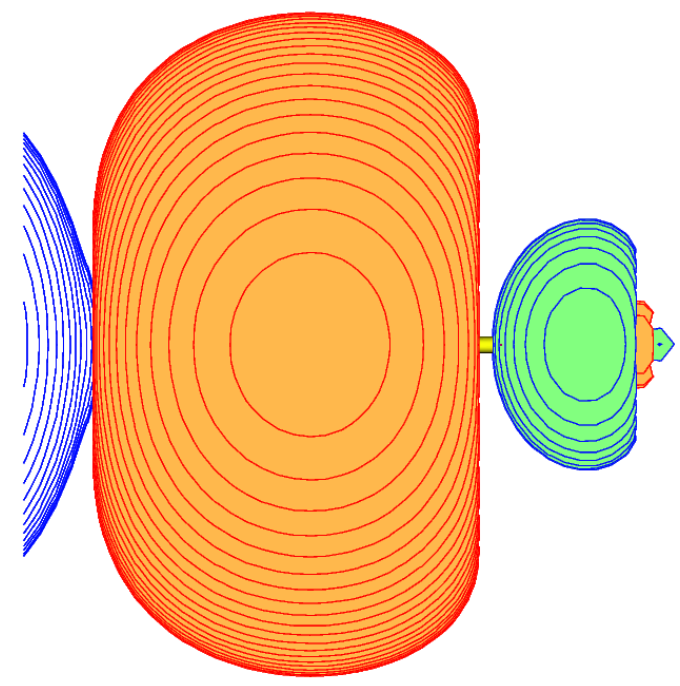

Figure 1: Molecular orbital of $\mathrm{MnF}$ in the ${ }^{1} \Sigma$ with contour value of 0.04 showing single bond character.

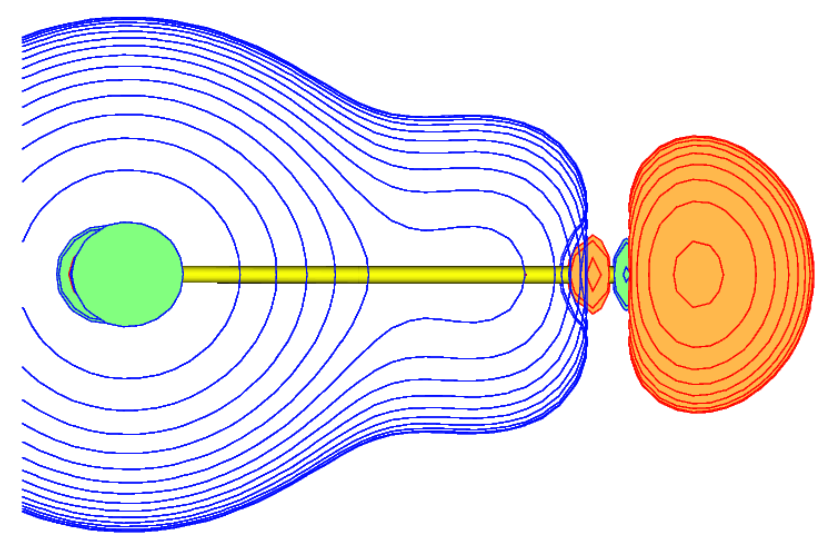

Figure 2: First bonding orbital for Ni(III)F in the ${ }^{1} \Sigma$ state with contour value 0.08 .

Observing $\mathrm{MnF}^{5} \Sigma$ yields similar results to the ${ }^{7} \Sigma$ state. Ligand field splitting is coherent with the theory, however, the orbitals occupations indicate that there is no bonding interaction between $\mathrm{F}$ and $\mathrm{Mn}$ in the ${ }^{5} \Sigma$ state.

At the experimental distance of 1.84 Åthe MRCI calculations reveal that ${ }^{5} \Sigma$ and ${ }^{1} \Sigma$ show bonding, however, ${ }^{7} \Sigma$ still does not show any bonding in the MOs.

For $\mathrm{Ni}(\mathrm{III}) \mathrm{F}$ forms much stronger bonds to fluorine than manganese alone, and it must be because $\mathrm{Ni}(\mathrm{III})$ can make more use of the donor properties of fluorine due to the positive charge. The stronger bonding behaviour of Ni(III)F compared to $\mathrm{MnF}$ is clearly seen from the orbital visualisations shown if Figure 2 to 4 In Ni(III)F, one can thus see a triple bond character from fluorine to $\mathrm{Ni}(\mathrm{III})$.

Pd(III)F should show intermediate behaviour compared to Ni(III)F and MnF. However, using the ECP yields a completely different shell. Thus, for better comparability using all electron basis sets (AE) are necessary.

\section{Discussion}

The Wigner-Witmer rules predict a ${ }^{7} \Sigma$ state for MnF and the state is indeed reported to be the ground state.[2, 3] However, assuming the state is the true ground state, it would mean there is no bond present, as there are essentially six unpaired electrons. However, a bond was observed experimentally in isolated matrix experiments for MnF[2]. Thus, 


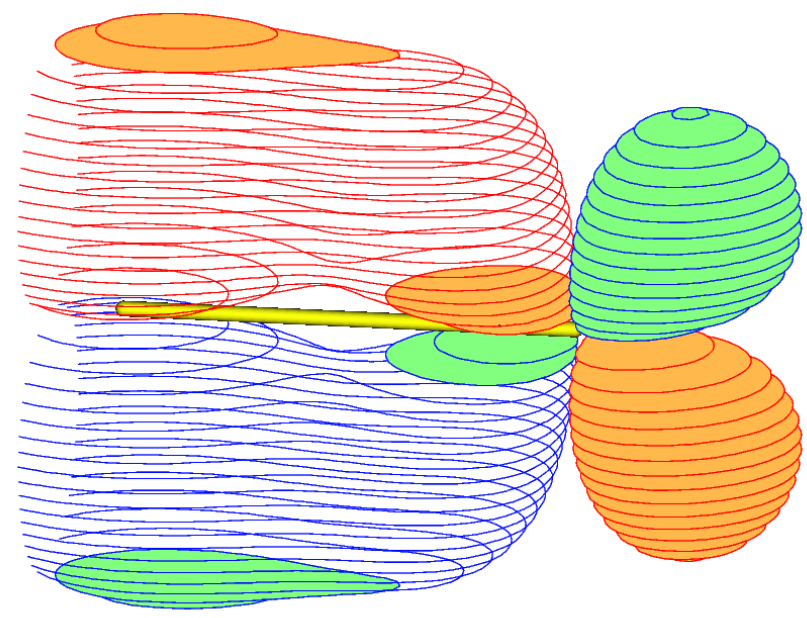

Figure 3: Second bonding orbital for Ni(III)F in the ${ }^{1} \Sigma$ state with a contour value of 0.08 .

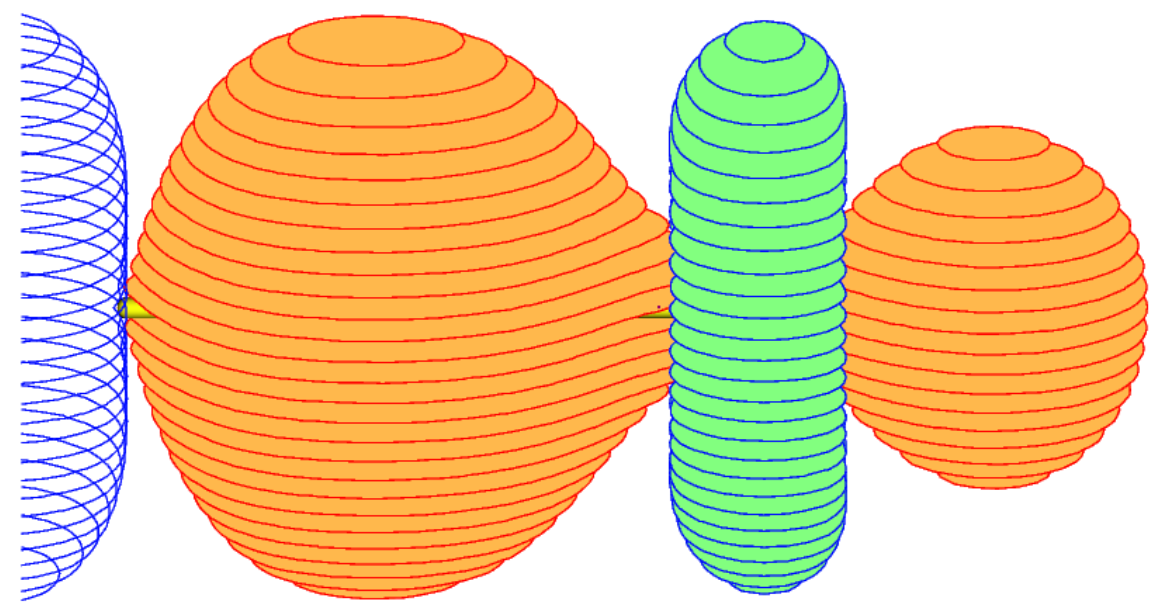

Figure 4: Third bonding orbital for $\mathrm{Ni}(\mathrm{III}) \mathrm{F}$ in the ${ }^{1} \Sigma$ state with contour value 0.08 .

rather the ${ }^{1} \Sigma$ or ${ }^{5} \Sigma$ is assumed as the ground state. Both would align with the weak IR stretching frequency observed[2]. Moreover, the calculated distance for the singlet state with HF level of theory is the closest to the experimental values from all different spin states investigated. The experimental value reported is 1.84 [9].

The trends should be verified further with, e.g., $\mathrm{Cu}(\mathrm{IV})$ or $\mathrm{Ag}(\mathrm{IV})$ or even $\mathrm{Zn}(\mathrm{V})$. These highly charged metal cations may, however, not be accessible to the experimental chemist any longer.

To verify the triple bond experimentally, it is suggested to try forming bonds to $\mathrm{Pd}(\mathrm{III}) \mathrm{F}$ or Ni(III)F. Subsequent experiments will be carried out to verify the hypothesis. In these experiments, the cation could be stabilized with weakly coordinating anions[10]. Moreover, $\mathrm{Ni}(\mathrm{III}) \mathrm{F}$ is synthetically easily accessible[11], such that observation of the bond should be possible with newly designed super acids[12, 13]. Spectroscopy should include PES, IR, UV/VIS and most importantly Mössbauer spectroscopy where possible. To provoke even higher bonding order such as quintuple bonds known from carbon chemistry metals such as $\mathrm{Mo}$, or $\mathrm{Cr}$ and their cations should be investigated and will be published in subsequent work. Different work is in the making focusing on lower bonding order and trends across halogens. Moreover, bonding to Lanthanides could provoke interesting bonding situations and will be studied as well. 


\section{References}

[1] G. S. Harbison, Journal of the American Chemical Society 2002, 124, 366-367.

[2] S. Chemate, N. Sekar, Journal of Fluorescence 2016, 26, 2063-2077.

[3] C. Koukounas, S. Kardahakis, A. Mavridis, Journal of Chemical Physics 2004, 120, 11500-11521.

[4] L. Bennett, B. Melchers, B. Proppe, Curta: A General-purpose High-Performance Computer at ZEDAT, Freie Universität Berlin, http://dx.doi.org/10.17169/refubium-26754, 2020.

[5] P. J. Knowles, N. C. Handy, 1989, 54, 75-83.

[6] H. J. Werner, P. J. Knowles, G. Knizia, F. R. Manby, M. Schütz, Wiley Interdisciplinary Reviews: Computational Molecular Science 2012, 2, 242-253.

[7] Gijs Schaftenaar, Molden, version 5.9.2, 2018.

[8] Molpro manual, https://www.molpro.net/manual/doku.php. (Accessed on 02/17/2022).

[9] P. M. Sheridan, L. M. Ziurys, Chemical Physics Letters 2003, 380, 632-646.

[10] M. C. Bayer, C. Kremser, C. Jessen, A. Nitzer, A. J. Kornath, Chemistry - A European Journal 2022, DOI 10.1002/chem.202104422.

[11] T. L. Court, M. F. A. Dove, J. Chem. Soc., Dalton Trans. 1973, 1995-1997.

[12] M. Hejda, D. Duvinage, E. Lork, A. Lyčka, Z. Černošek, J. Macháček, S. Makarov, S. Ketkov, S. Mebs, L. Dostál, J. Beckmann, Chemistry - A European Journal 2021, 27, 14577-14581.

[13] L. Greb, Chemistry - A European Journal 2018, 24, 17881-17896. 